\title{
Net clinical benefit of anticoagulation therapy in the elderly patients with atrial fibrillation
}

\author{
Lorenzo Palleschi', Eleonora Nunziata² \\ 1 Unit of Geriatrics, AO S. Giovanni-Addolorata, Rome \\ 2 Unit of Medicine, Asl RM4, Rome, Italy
}

\begin{abstract}
Old age remains one of the strongest risk factors for stroke in patients with atrial fibrillation (AF). Oral anticoagulation (OAC) is the most effective way to prevent thromboembolic disease in patients with atrial fibrillation (AF). Until few years ago, aspirin and vitamin-K antagonists (VKAs) were the primary agents used to prevent thromboembolic disease in patients with AF. The approval of non-vitamin $\mathrm{K}$ oral anticoagulants (NOACs) has now expanded the range of therapeutic agents available to providers. In our paper, we highlight practical considerations regarding the selection and use of OAC in older adults to aid clinical decision making.
\end{abstract}

\section{Management goals}

The aim of oral anticoagulation (OAC) is to prevent the devastating consequences of stroke in older adults with AF while minimizing complications from treatment. Older patients are at increased risk of bleeding complications, adding to the complexity of their treatment. The decision to use antithrombotic therapy in an individual patient with $\mathrm{AF}$ requires an estimate of the baseline stroke risk without treatment and the risk of bleeding (especially intracranial hemorrhage [ICH]) with treatment. Periodic reevaluation of the patient's stroke and bleeding risk is essential [1,2].

Stroke risk in individual patients with AF varies from less than $1 \%$ per year to more than $18 \%$ per year and depends on the presence, number, and relative predictive strength of different clinical risk fac-

Corresponding author: Eleonora Nunziata, Unit of Medicine, Asl RM4, Via Terme di Traiano 39/A, 00053 Civitavecchia, Rome, Italy.

E-mail: ele.nunziata@gmail.com

Key words: Atrial fibrillation; non-vitamin K antagonist oral anticoagulation; warfarin; elderly; stroke; hemorrhage; frailty.

Received for publication: 24 April 2018

Accepted for publication: 11 May 2018

CCopyright L. Palleschi and E. Nunziata, 2018

Tipografia PI-ME Editrice, Italy

Monaldi Archives for Chest Disease 2018; 88:961

doi: 10.4081/monaldi.2018.961

This article is distributed under the terms of the Creative Commons Attribution Noncommercial License (by-nc 4.0) which permits any noncommercial use, distribution, and reproduction in any medium, provided the original author(s) and source are credited. tors for stroke. For patients with nonvalvular atrial fibrillation (NVAF), the 4 strongest stroke predictors are prior stroke and/or TIA (relative risk [RR] 5 2.5), hypertension (RR 5 2.0), diabetes mellitus (RR 5 1.7), and age (RR 51.5 per decade) [2].

Validated stroke and bleeding risk stratification schemes, such as the CHA2DS2-VASC score (quantified by the Congestive Heart Failure, Hypertension, Age $\geq 75$ doubled, Diabetes, Stroke doubled - Vascular disease, Age 65-74 and female Sex) and the HAS-BLED score (quantified by Hypertension, Abnormal renal/iver function, Stroke, Bleeding history or predisposition, Labile international normalized ratio, Elderly $>65$ years, Drugs/alcohol concomitantly), should be used to estimate stroke risk and guide anticoagulation decisions in older adults with AF [3]. Bleeding risk scores, such as HAS-BLED, should not be used to exclude patients from the use of oral anticoagulation (OAC) but rather to identify modifiable bleeding risk factors that can be managed to reduce a patient's risk of bleeding from anticoagulation [3].

\section{Oral anticoagulation with vitamin-K antagonists (VKAs) in older patients}

The elderly presents a unique challenge in antithrombotic management because of the frequency of associated comorbidities that increase the risk of both stroke and bleeding: multiple drug therapy, concerns about compliance and cognitive impairment, low body weight, increased risk of falls, and decreased renal clearance of medications.

Compared with no treatment, warfarin decreases the risk of stroke by approximately two-thirds and death by one-fourth [4]. Despite compelling evidence of the benefits of warfarin on stroke prevention, it remains underutilized, with estimates that less than half of eligible patients with AF without contraindications are receiving treatment $[5,6]$.

The challenges of warfarin are well known and include unpredictable pharmacodynamics and pharmacokinetics, narrow therapeutic window, and numerous drug-drug and food-drug interactions, which necessitate frequent laboratory monitoring and dose adjustments. Patient and physician fears of bleeding also contribute to suboptimal warfarin utilization. Physicians are less likely to use anticoagulation in older patients, even in the absence of contraindications, because of the perception that the risks of treatment outweigh the benefits [6,7]. Each advancing decade of life is associated with a $14 \%$ reduction in warfarin use, independent of other risk factors for stroke [8]. Results from the ATA AF multicenter observational study demonstrated that in more than half of the patients the factor that most influenced the nonprescription of OAT was age [9]. Nevertheless, a recent study that took place within the last decade observed a substantial increase in anticoagulation utilization in survivors of acute brain ischemia with atrial fibrillation. This change was mainly driven by greater utilization of anticoagulation in subgroups with traditional clinical barriers to anticoagulation use, indicating a shift in physicians' perceptions of the risk-benefit ratio of anticoagulation [10]. 
Based on the need to balance the risks of ischemic TE with the risks of fatal and disabling major bleeding, prospective cohort studies of patients with AF have assessed the net clinical benefit of warfarin, which has been established as the annual rate of ischemic strokes prevented by warfarin minus the annual rate of ICH caused by warfarin, the latter multiplied by a factor of 1.5 to reflect the greater clinical severity of ICH than ischemic stroke [11]. Overall, the impact of warfarin is much higher on TE risk than on ICH risk. Owing to increasing risk for stroke with aging, the net clinical benefit of warfarin improves progressively with advancing age and is greatest for patients with AF 85 years and older. Net clinical benefit of warfarin also improves progressively with major bleeding risk as measured by the HAS-BLED score; that is, warfarin $\mathrm{OAC}$ decreases ischemic stroke rate more than it increases ICH rate at all levels of bleeding risk [11].

In the elderly discontinuing warfarin after a diagnosis of dementia is associated with a significant increase in stroke and mortality [12]. A recent retrospective cohort study that included 1,405 individuals aged $>75$ years with atrial fibrillation and ischemic stroke after surviving hospitalization has shown that despite very high stroke risk, more than $40 \%$ of participants were not discharged with an OAC. Dominant reasons included risk of falling, poor prognosis, old age, and dementia. These individuals' high 1-year mortality rate confirmed their high level of comorbidity [13].

\section{Non-vitamin K-dependent oral anticoagulants (NOACs) in older patients}

The US Food and Drug Administration and European Medicines Agency have approved 4 NOACs (dabigatran, rivaroxaban, apixaban and edoxaban) for use in AF. These newer drugs provide clinicians with additional therapeutic options to prevent stroke in AF and lack many of the limitations inherent to warfarin. They have predictable pharmacodynamic and pharmacokinetic profiles, a wide therapeutic window, shorter half-life with rapid onset/offset of action, fewer drug-drug interactions, few to no food-drug interactions, no need for routine laboratory monitoring, and fixed dosing schedules.

Dabigatran is an oral direct thrombin inhibitor, whereas rivaroxaban, apixaban, and edoxaban are factor Xa inhibitors. Each of these drugs is either noninferior or superior to warfarin for stroke prevention, with markedly less intracranial bleeding. Dabigatran, rivaroxaban, and edoxaban caused higher rates of GI bleeding than warfarin. NOACs are associated with an approximately $12 \%$ decrease in mortality compared with warfarin, a $20 \%$ decrease in stroke or systemic embolus, and a $50 \%$ reduction in ICH. The net clinical benefit of NOACs is not yet as clearly established as it is for warfarin.

Although there are no randomized controlled trials (RCTs) that have enrolled exclusively older patients with AF, the available evidence suggests that the safety and efficacy of NOACs in older adults is similar to the overall study population [14]. A meta-analysis of randomized trials of NOACs in patients 75 years or older showed significantly lower rates of stroke and systemic embolus than conventional treatment, with no increased risk of bleeding [15].

In the rivaroxaban once daily oral direct factor Xa inhibition compared with vitamin $\mathrm{K}$ antagonism for prevention of stroke and embolism trial in atrial fibrillation (ROCKET AF), the risk of any major bleeding was similar among patients who received rivaroxaban or warfarin, regardless of age, but intracranial bleeding was less with rivaroxaban.

In apixaban for reduction in stroke and other thromboembolic events in atrial fibrillation (ARISTOTLE), the overall risk of stroke, death, and major bleeding increased with aging, but apixaban was more effective than warfarin in reducing these outcomes, irrespective of age.
Owing to increasing risk of stroke as patients age, the absolute benefit of NOACs over warfarin was greatest in the elderly. In contrast to rivaroxaban and apixaban, in the randomized evaluation of long-term anticoagulation therapy (RE-LY), there was a highly significant interaction between treatment and age for major bleeding. In patients younger than 75 years, both doses of dabigatran were associated with a lower risk of major bleeding than warfarin. However, patients 75 years or older experienced similar rates of major extracranial bleeding compared with warfarin with dabigatran at a dose of $110 \mathrm{mg}$ twice daily, but a trend toward higher risk of extracranial bleeding with the dose of $150 \mathrm{mg}$ twice daily. ICH was reduced with both doses of dabigatran compared with warfarin, regardless of age. These data suggest that the safety of $\mathrm{Xa}$ inhibitors is less dependent on age than dabigatran, with one possible explanation being that they are less dependent on renal clearance than dabigatran.

NOACs have not yet been robustly studied in patients with severe renal impairment and are not recommended in patients with end-stage renal disease or who are undergoing hemodialysis. The incidence of renal impairment increases with age, and renal impairment increases the risk of both TE and bleeding complications in patients with AF, independent of other risk factors. Because AF is primarily a disease of the elderly, these two conditions often coexist, leading to significant concerns about bleeding risk.

A significant minority (almost 1 in 8 ) of U.S. patients in the community received NOAC doses inconsistent with labeling. NOAC over- and underdosing are associated with increased risk for adverse events [16].

Despite the fact that NOACs seem to be at least as safe as warfarin in the short-term and reduce the risk of intracranial bleeding, longterm data are still needed. There may also be subtle differences between the NOACs in rates of ischemic stroke, myocardial infarction, bleeding, and death, but the lack of head-to-head trials makes this difficult to discern. Nevertheless, when considered overall, the significant decrease in ICH, fixed dosing schedules, lack of routine laboratory monitoring, and fewer drug-drug interactions make the NOACs an attractive alternative to warfarin for some older patients.

\section{How to improve the OAT risk assessment in elderly?}

Anticoagulation is particularly complex in the frail elderly patient, who presents additional risk factors affecting both the efficacy and safety of thromboembolic prevention. Main clinical trials rarely include frail elderly patients and, consequently, the guidelines for treatment do not provide instruction for their management. In the absence of clear advice for this class of patients, physicians should identify areas to be taken into account both before starting and when discontinuing anticoagulation: comorbidities, polypharmacotherapy, adherence, cognitive impairment, mobility and monitoring barriers, nutritional status and swallowing disorders, risk of falls, and reduced life expectancy.

The Frail-AF Study investigated the effect of thromboembolic risk, bleeding risk, and frailty on the anticoagulation status in 682 octogenarians hospitalized with AF. Consumption of warfarin or a new oral anticoagulant was documented. Medical record data were used to determine each patient's frailty status using the Clinical Frailty Scale (CFS: functional autonomy, mobility, assistance in ADL, cognitive status, nutrition, polypharmacy, multimorbidity) and to evaluate the risk of stroke and bleeding using CHADS2 and HAS-BLED. This study suggested a higher prevalence of appropriate anticoagulation therapy among octogenarians with AF than reported in previous studies. It seems that several validated frailty scales can optimize the use of anticoagulants in this high-risk population [17]. 
A retrospective observational study investigated the relationship between warfarin treatment and different levels of all-cause mortality risk assessed using the Multidimensional Prognostic Index (MPI) based on information collected using the Standardized Multidimensional Assessment Schedule for Adults and Aged Persons (SVaMA) in communitydwelling older adults with atrial fibrillation (AF). Participants were classified as being at mild (MPI-SVaMA-1), moderate (MPI-SVaMA-2), or severe (MPI-SVaMA-3) risk of mortality using the MPI-SVaMA, a validated prognostic tool based on age, sex, comorbidity, cognitive status, mobility and functional disability, pressure sore risk, and social support. In conclusion, community-dwelling older adults with AF benefitted from anticoagulation in terms of lower all-cause mortality over a mean follow-up of 2 years, regardless of poor health and functional condition [18].

Some authors suggest a multidimensional algorithm covering both a standard ischemic and bleeding risk assessment and an additional anticoagulation-focused frailty assessment. This is of particular relevance given the recent introduction of the oral direct inhibitors, as they are likely to widen the treatment options for the frail elderly. Depending on which aspect of frailty is present, anticoagulation could now be tailored accordingly [19].

\section{Future considerations and summary}

In summary, atrial fibrillation is a common condition in the elderly and the incidence of thromboembolic events secondary to atrial fibrillation increases with age. Antithrombotic therapy effectively prevents stroke and systemic embolism but also exposes patients to the risk of bleeding. Recently approved NOACs may offer some selected advantages for the prevention of stroke and systemic embolism in older patients with AF.

Because the risk of bleeding also increases with age, clinicians tend to withhold anticoagulation in the elderly. The use of guideline-recommended stroke scoring systems, in combination with a bleeding risk factor assessment, can help guide providers in determining the net clinical benefit of antithrombotic therapy in older adults with AF. To improve anticoagulation decisions and outcomes in older adults with AF, future studies to test usefulness of a geriatric comprehensive assessment, besides the scores addressing risk of thromboembolic and hemorrhagic events, may further elucidate the net clinical benefit of NOACs in the elderly and help guide more individualized AC selection and management.

\section{References}

1. Kirchhof P, Benussi S, Kotecha D, et al. 2016 ESC Guidelines for the management of atrial fibrillation developed in collaboration with EACTS. Eur Heart J 2016;37:2893-962.

2. January CT, Wann LS, Alpert JS, et al. 2014 AHA/ACC/HRS guideline for the management of patients with atrial fibrillation: a report of the American College of Cardiology/American Heart Association Task Force on practice guidelines and the Heart Rhythm Society. J Am Coll Cardiol 2014;64:2246-80.
3. Friberg L, Rosenqvist M, Lip GY. Evaluation of risk stratification schemes for ischaemic stroke and bleeding in 182678 patients with atrial fibrillation: the Swedish Atrial Fibrillation cohort study. Eur Heart J 2012;33:1500-10.

4. Hart RG, Pearce LA, Aguilar MI. Meta-analysis: antithrombotic therapy to prevent stroke in patients who have nonvalvular atrial fibrillation. Ann Intern Med 2007;146:857-67.

5. Ogilvie IM, Newton N, Welner SA, et al. Underuse of oral anticoagulants in atrial fibrillation: a systematic review. Am J Med 2010;123:638-45.

6. Lip GY1, Lane DA. Stroke prevention with oral anticoagulation therapy in patients with atrial fibrillation-focus on the elderly. Circ J 2013;77:1380-8.

7. Pugh D, Pugh J, Mead GE. Attitudes of physicians regarding anticoagulation for atrial fibrillation: a systematic review. Age Ageing 2011;40:675-83.

8. Brophy MT, Snyder KE, Gaehde S, et al. Anticoagulant use for atrial fibrillation in the elderly. J Am Geriatr Soc 2004;52:1151-6.

9. Di Pasquale G, Mathieu G, Maggioni AP, et al. Current presentation and management of 7148 patients with atrial fibrillation in cardiology and internal medicine hospital centers: the ATA AF study. Int J Cardiol 2013;167:2895-903.

10. Kirchhof P, Benussi S, Kotecha D, et al. 2016 ESC Guidelines for the management of atrial fibrillation developed in collaboration with EACTS. Eur Heart J 2016;37:2893-962.

11. Friberg L, Rosenqvist M, Lip GY. Net clinical benefit of warfarin in patients with atrial fibrillation: a report from the Swedish Atrial Fibrillation cohort study. Circulation 2012;125:2298-307.

12. Orkaby AR, Ozonoff A., Reisman JI, et al. Continued use of warfarin in veterans with atrial fibrillation after dementia diagnosis. J Am Geriatr Soc 2017;65:249-56.

13. McGrath ER, Go AS, Chang Y, et al. Use of Oral Anticoagulant Therapy in Older Adults with Atrial Fibrillation After Acute Ischemic Stroke. J Am Geriatr Soc 2017;65:241-8.

14. Deedwania PC. New oral anticoagulants in elderly patients with atrial fibrillation. Am J Med 2013;126:289-96.

15. Sardar P, Chatterjee S, Chaudhari S, et al. New oral anticoagulants in elderly adults: evidence from a meta-analysis of randomized trials. J Am Geriatr Soc 2014;62:857-64.

16. Steinberg B, Shrader P, Thomas L, et al. Off-label dosing of nonvitamin $\mathrm{K}$ antagonist oral anticoagulants and adverse outcomes: The ORBIT-AF II Registry. J Am Coll Cardiol 2016 20;68:2597-604.

17. Lefebvre MC, St-Onge M, Glazer-Cavanagh M, et al. The effect of bleeding risk and frailty status on anticoagulation patterns in octogenarians with atrial fibrillation: The FRAIL-AF Study. Can J Cardiol 2016;32:169-76.

18. Pilotto A, Gallina P, Copetti M, et al. Warfarin treatment and allcause mortality in community-dwelling older adults with atrial fibrillation: A retrospective observational study. J Am Geriatr Soc 2016;64:1416-24.

19. Granziera S, Cohen AT, Nante G, et al. Thromboembolic prevention in frail elderly patients with atrial fibrillation: a practical algorithm. J Am Med Dir Assoc 2015;16:358-64. 